\title{
Global behavior of the solutions of some difference equations
}

Elmetwally M Elabbasy ${ }^{1 *}$, Hamdy A El-Metwally ${ }^{2,4}$ and Elsayed M Elsayed ${ }^{3,4}$

* Correspondence:

emelabbasy@mans.edu.eg

'Mathematics Department, Faculty

of Science, Mansoura University,

Mansoura 35516, Egypt

Full list of author information is

available at the end of the article

\section{Abstract}

In this article we study the difference equation

$$
x_{n+1}=\frac{a x_{n-l} x_{n-k}}{b x_{n-p}-c x_{n-q}}, n=0,1, \ldots,
$$

where the initial conditions $x_{-r}, X_{-r+1}, X_{-r+2}, \ldots, x_{0}$ are arbitrary positive real numbers, $r=$ $\max \{1, k, p, q\}$ is nonnegative integer and $a, b, c$ are positive constants: Also, we study some special cases of this equation.

Keywords: Stability, Solutions of the difference equations

\section{Introduction}

The purpose of this article is to investigate the global attractivity of the equilibrium point, and the asymptotic behavior of the solutions of the following difference equation

$$
x_{n+1}=\frac{a x_{n-l} x_{n-k}}{b x_{n-p}-c x_{n-q}}, n=0,1, \ldots
$$

where the initial conditions $x_{-r}, x_{-r+1}, x_{-r+2}, \ldots, x_{0}$ are arbitrary positive real numbers, $r$ $=\max \{l, k, p, q\}$ is nonnegative integer and $a, b, c$ are positive constants: Moreover, we obtain the form of the solution of some special cases of Equation 1 and some numerical simulations to the equation are given to illustrate our results.

Let us introduce some basic definitions and some theorems that we need in the sequel.

Let $I$ be some interval of real numbers and let

$$
f: I^{k+1} \rightarrow I,
$$

be a continuously differentiable function. Then for every set of initial conditions $x_{-k}$, $x_{-k+1}, \ldots, x_{0} \in I$, the difference equation

$$
x_{n+1}=f\left(x_{n}, x_{n-1}, \ldots, x_{n-k}\right), n=0,1, \ldots,
$$

has a unique solution $\left\{x_{n}\right\}_{n=-k}^{\infty}[1]$.

A point $\bar{x} \in I$ is called an equilibrium point of Equation 2 if

$$
\bar{x}=f(\bar{x}, \bar{x}, \ldots, \bar{x}) .
$$


That is, $x_{n}=\bar{x}$ for $n \geq 0$, is a solution of Equation 2, or equivalently, $\bar{x}$ is a fixed point of $f$.

Definition 1 (Stability)

(i) The equilibrium point $\bar{x}$ of Equation 2 is locally stable if for every $\varepsilon>0$, there exists $\delta>0$ such that for all $x_{-k}, x_{-k+1}, \ldots, x_{-1}, x_{0} \in I$ with

$$
\left|x_{-k}-\bar{x}\right|+\left|x_{-k+1}-\bar{x}\right|+\cdots+\left|x_{0}-\bar{x}\right|<\delta,
$$

we have

$$
\left|x_{n}-\bar{x}\right|<\varepsilon \text { for all } n \geq-k .
$$

(ii) The equilibrium point $\bar{x}$ of Equation 2 is locally asymptotically stable if $\bar{x}$ is locally stable solution of Equation 2 and there exists $\gamma>0$, such that for all $x_{-k}, x_{-k+1}, \ldots$, $x_{-1}, x_{0} \in I$ with

$$
\left|x_{-k}-\bar{x}\right|+\left|x_{-k+1}-\bar{x}\right|+\ldots+\left|x_{0}-\bar{x}\right|<\gamma,
$$

we have

$$
\lim _{n \rightarrow \infty} x_{n}=\bar{x} .
$$

(iii) The equilibrium point $\bar{x}$ of Equation 2 is global attractor if for all $x_{-k}, x_{-k+1}, \ldots, x_{-1}$, $x_{0} \in I$, we have

$$
\lim _{n \rightarrow \infty} x_{n}=\bar{x} .
$$

(iv) The equilibrium point $\bar{x}$ of Equation 2 is globally asymptotically stable if $\bar{x}$ is locally stable and $\bar{x}$ is also a global attractor of Equation 2 .

(v) The equilibrium point $\bar{x}$ of Equation 2 is unstable if $\bar{x}$ is not locally stable.

The linearized equation of Equation 2 about the equilibrium $\bar{x}$ is the linear difference equation

$$
y_{n+1}=\sum_{i=0}^{k} \frac{\partial f\left(\bar{x}, \bar{x}_{1} \ldots, \bar{x}\right)}{\partial x_{n-i}} y_{n-i} .
$$

\section{Theorem A [2]}

Assume that $p, q \in R$ and $k \in\{0,1,2, \ldots\}$. Then

$$
|p|+|q|<1,
$$

is a sufficient condition for the asymptotic stability of the difference equation

$$
x_{n+1}+p x_{n}+q x_{n-k}=0, n=0,1, \ldots
$$

Remark 1 Theorem A can be easily extended to a general linear equations of the form

$$
x_{n+k}+p_{1} x_{n+k-1}+\ldots+p_{k} x_{n}=0, n=0,1, \ldots,
$$

where $p_{1}, p_{2}, \ldots, p_{k} \in R$ and $k \in\{1,2, \ldots\}$. Then Equation 4 is asymptotically stable provided that

$$
\sum_{i=1}^{k}\left|p_{i}\right|<1
$$




\section{Definition 2}

(Fibonacci Sequence) The sequence $\left\{F_{m}\right\}_{m=0}^{\infty}=\{1,2,3,5,8,13, \ldots\}$ i.e. $F_{m}=F_{m-1}+F_{m-2}$, $m \geq 0, F_{-2}=0, F_{-1}=1$ is called Fibonacci Sequence.

The nature of many biological systems naturally leads to their study by means of a discrete variable. Particular examples include population dynamics and genetics. Some elementary models of biological phenomena, including a single species population model, harvesting of fish, the production of red blood cells, ventilation volume and blood $\mathrm{CO}_{2}$ levels, a simple epidemics model and a model of waves of disease that can be analyzed by difference equations are shown in [3]. Recently, there has been interest in so-called dynamical diseases, which correspond to physiological disorders for which a generally stable control system becomes unstable. One of the first papers on this subject was that of Mackey and Glass [4]. In that paper they investigated a simple first order difference-delay equation that models the concentration of blood-level $\mathrm{CO}_{2}$. They also discussed models of a second class of diseases associated with the production of red cells, white cells, and platelets in the bone marrow.

The study of the nonlinear rational difference equations of a higher order is quite challenging and rewarding, and the results about these equations offer prototypes towards the development of the basic theory of the global behavior of nonlinear difference equations of a big order, recently, many researchers have investigated the behavior of the solution of difference equations for example: Elabbasy et al. [5] investigated the global stability, periodicity character and gave the solution of special case of the following recursive sequence

$$
x_{n+1}=a x_{n}-\frac{b x_{n}}{c x_{n}-d x_{n-1}} .
$$

Elabbasy et al. [6] investigated the global stability, boundedness, periodicity character and gave the solution of some special cases of the difference equation

$$
x_{n+1}=\frac{\alpha x_{n-k}}{\beta+\gamma \prod_{i=0}^{k} x_{n-i}} .
$$

Elabbasy et al. [7] investigated the global stability character, boundedness and the periodicity of solutions of the difference equation

$$
x_{n+1}=\frac{\alpha x_{n}+\beta x_{n-1}+\gamma x_{n-2}}{A x_{n}+B x_{n-1}+C x_{n-2}} .
$$

El-Metwally et al. [8] investigated the asymptotic behavior of the population model:

$$
x_{n+1}=\alpha+\beta x_{n-1} e^{-x_{n}}
$$

where $\alpha$ is the immigration rate and $\beta$ is the population growth rate.

Yang et al. [9] investigated the invariant intervals, the global attractivity of equilibrium points and the asymptotic behavior of the solutions of the recursive sequence

$$
x_{n+1}=\frac{a x_{n-1}+b x_{n-2}}{c+d x_{n-1} x_{n-2}} .
$$


Cinar $[10,11]$ has got the solutions of the following difference equations

$$
x_{n+1}=\frac{x_{n-1}}{1+a x_{n} x_{n-1}}, x_{n+1}=\frac{x_{n-1}}{-1+a x_{n} x_{n-1}} .
$$

Aloqeili [12] obtained the form of the solutions of the difference equation

$$
x_{n+1}=\frac{x_{n-1}}{a-x_{n} x_{n-1}} .
$$

Yalçinkaya [13] studied the following nonlinear difference equation

$$
x_{n+1}=\alpha+\frac{x_{n-m}}{x_{n}^{k}} .
$$

For some related work see [1-29].

The article proceeds as follows. In Sect. 2 we show that when $2 a|b-c|+a(b+c)<$ $(b-c)^{2}$, then the equilibrium point of Equation 1 is locally asymptotically stable. In Sect. 3 we prove that the equilibrium point of Equation 1 is global attractor. In Sect. 4 we give the solutions of some special cases of Equation 1 and give a numerical examples of each case and draw it by using Matlab 6.5.

\section{Local stability of Equation 1}

In this section we investigate the local stability character of the solutions of Equation 1. Equation 1 has a unique positive equilibrium point and is given by

$$
\bar{x}=\frac{a \bar{x}^{2}}{b \bar{x}-c \bar{x}},
$$

if $a \neq b-c, b \neq c$, then the unique equilibrium point is $\bar{x}=0$.

Let $f:(0, \infty)^{4} \rightarrow(0, \infty)$ be a function defined by

$$
f(u, v, w, s)=\frac{a u v}{b w-c s} .
$$

Therefore, it follows that

$$
\begin{aligned}
& f_{u}(u, v, w, s)=\frac{a v}{(b w-c s)}, \quad f_{v}(u, v, w, s)=\frac{a u}{(b w-c s)}, \\
& f_{w}(u, v, w, s)=\frac{-b a u v}{(b w-c s)^{2}}, \quad f_{s}(u, v, w, s)=\frac{c a u v}{(b w-c s)^{2}},
\end{aligned}
$$

we see that

$$
\begin{aligned}
& f_{u}(\bar{x}, \bar{x}, \bar{x}, \bar{x})=\frac{a}{(b-c)}, \quad f_{v}(\bar{x}, \bar{x}, \bar{x}, \bar{x})=\frac{a}{(b-c)} \\
& f_{w}(\bar{x}, \bar{x}, \bar{x}, \bar{x})=\frac{-a b}{(b-c)^{2}}, \quad f_{s}(\bar{x}, \bar{x}, \bar{x}, \bar{x})=\frac{a c}{(b-c)^{2}} .
\end{aligned}
$$

The linearized equation of Equation 1 about $\bar{x}$ is

$$
y_{n+1}+\frac{a}{(b-c)} y_{n-1}+\frac{a}{(b-c)} y_{n-k}-\frac{a b}{(b-c)^{2}} y_{n-p}+\frac{a c}{(b-c)^{2}} y_{n-q}=0 .
$$




\section{Theorem 1}

Assume that

$$
a(3 \zeta-\eta)<(b-c)^{2}
$$

where $\zeta=\max \{b, c\}, \eta=\min \{b, c\}$. Then the equilibrium point of Equation 1 is locally asymptotically stable.

Proof: It is follows by Theorem A that Equation 6 is asymptotically stable if

$$
\left|\frac{a}{(b-c)}\right|+\left|\frac{a}{(b-c)}\right|+\left|\frac{a b}{(b-c)^{2}}\right|+\left|\frac{a b}{(b-c)^{2}}\right|<1,
$$

or

$$
\left|\frac{2 a}{(b-c)}\right|+\left|\frac{a(b+c)}{(b-c)^{2}}\right|<1,
$$

and so

$$
2 a|b-c|+a(b+c)<(b-c)^{2} .
$$

The proof is complete.

\section{Global attractivity of the equilibrium point of Equation 1}

In this section we investigate the global attractivity character of solutions of Equation 1.

We give the following two theorems which is a minor modification of Theorem A.0.2 in [1].

\section{Theorem 2}

Let $[a, b]$ be an interval of real numbers and assume that

$$
f:[a, b]^{k+1} \rightarrow[a, b]
$$

is a continuous function satisfying the following properties:

(i) $f\left(x_{1}, x_{2}, \ldots ., x_{k+1}\right)$ is non-increasing in one component (for example $\left.x_{t}\right)$ for each $x_{r}(r$ $\neq t)$ in $[a, b]$ and non-decreasing in the remaining components for each $x_{t}$ in $[a, b]$.

(ii) If $(m, M) \in[a, b] \times[a, b]$ is a solution of the system $M=f(M, M, \ldots, M, m, M, \ldots, M, M)$ and $m=f(m, m, \ldots, m, M, m, \ldots m, m)$ implies

$$
m=M \text {. }
$$

Then Equation 2 has a unique equilibrium $\bar{x} \in[a, b]$ and every solution of Equation 2 converges to $\bar{x}$

Proof: Set

$$
m_{0}=a \text { and } M_{0}=b,
$$

and for each $i=1,2, \ldots$ set

$$
m_{i}=f\left(m_{i-1}, m_{i-1}, \ldots, m_{i-1}, M_{i-1}, m_{i-1}, \ldots, m_{i-1}, m_{i-1}\right),
$$

and

$$
M_{i}=f\left(M_{i-1}, M_{i-1}, \ldots, M_{i-1}, m_{i-1}, M_{i-1}, \ldots, M_{i-1}, M_{i-1}\right) .
$$


Now observe that for each $i \geq 0$,

$$
a=m_{0} \leq m_{1} \leq \ldots \leq m_{i} \leq \ldots \leq M_{i} \leq \ldots \leq M_{1} \leq M_{0}=b,
$$

and

$$
m_{i} \leq x_{p} \leq M_{i} \text { for } p \geq(k+1) i+1 .
$$

Set

$$
m=\lim _{x \rightarrow \infty} m_{i} \text { and } M=\lim _{i \rightarrow \infty} M_{i} .
$$

Then

$$
M \geq \lim _{i \rightarrow \infty} \sup x_{i} \geq \lim \inf _{i \rightarrow \infty} x_{i} \geq m
$$

and by the continuity of $f$,

$M=f(M, M, \ldots, M, m, M, \ldots, M, M)$ and $m=f(m, m, \ldots, m, M, m, \ldots m, m)$.

In view of (ii),

$$
m=M=\bar{x},
$$

from which the result follows.

\section{Theorem 3}

Let $[a, b]$ be an interval of real numbers and assume that

$$
f:[a, b]^{k+1} \rightarrow[a, b]
$$

is a continuous function satisfying the following properties:

(i) $f\left(x_{1}, x_{2}, \ldots, x_{k+1}\right)$ is non-increasing in one component (for example $x_{t}$ ) for each $x_{r}(r$

$\neq t$ ) in $[a, b]$ and non-increasing in the remaining components for each $x_{t}$ in $[a, b]$.

(ii) If $(m, M) \in[a, b] \times[a, b]$ is a solution of the system

$M=f(m, m, \ldots, m, M, m, \ldots m, m)$ and $m=f(M, M, \ldots, M, m, M, \ldots, M, M)^{\prime}$ implies

$$
m=M \text {. }
$$

Then Equation 2 has a unique equilibrium $\bar{x} \in[a, b]$ and every solution of Equation 2 converges to $\bar{x}$

Proof: As the proof of Theorem 2 and will be omitted.

\section{Theorem 4}

The equilibrium point $\bar{x}$ of Equation 1 is global attractor if $c \neq a$.

Proof: Let $p, q$ are a real numbers and assume that $f:[p, q]^{4} \rightarrow[p, q]$ be a function defined by Equation 5 , then we can easily see that the function $f(u, v, w, s)$ increasing in $s$ and decreasing in $w$.

Case (1) If $b w-c s>0$, then we can easily see that the function $f(u, v, w, s)$ increasing in $u, v, s$ and decreasing in $w$.

Suppose that $(m, M)$ is a solution of the system $M=f(m, m, M, m)$ and $M=f(M, M, m, M)$.

Then from Equation 1, we see that

$$
m=\frac{a m^{2}}{b M-c m}, \quad M=\frac{a M^{2}}{b m-c M},
$$




$$
b M=c m+a m, \quad b m=c M+a M,
$$

then

$$
(M-m)(b+c+a)=0 .
$$

Thus

$$
M=m \text {. }
$$

It follows by Theorem 2 that $\bar{x}$ is a global attractor of Equation 1 and then the proof is complete.

Case (2) If $b w$-cs $<0$, then we can easily see that the function $f(u, v, w, s)$ decreasing in $u, v, w$ and increasing in $s$.

Suppose that $(m, M)$ is a solution of the system

$M=f(m, m, m, M)$ and $m=f(M, M, M, m)$.

Then from Equation 1, we see that

$$
\begin{aligned}
& M=\frac{a m^{2}}{b m-c M^{2}}, \quad m=\frac{a M^{2}}{b M-c m^{\prime}} \\
& b m M-c M^{2}=a m^{2}, b m M-c m^{2}=a M^{2},
\end{aligned}
$$

then

$$
\left(M^{2}-m^{2}\right)(c-a)=0, \quad a \neq c .
$$

Thus,

$$
M=m \text {. }
$$

It follows by the Theorem 3 that $\bar{x}$ is a global attractor of Equation 1 and then the proof is complete.

\section{Special cases of Equation 1}

\subsection{Case (1)}

In this section we study the following special case of Equation 1

$$
x_{n+1}=\frac{x_{n} x_{n-1}}{x_{n}-x_{n-1}}
$$

where the initial conditions $x_{-1}, x_{0}$ are arbitrary positive real numbers.

\section{Theorem 5}

Let $\left\{x_{n}\right\}_{n=-1}^{\infty}$ be a solution of Equation 7. Then for $n=0,1, \ldots$

$$
x_{n}=\frac{(-1)^{n} h k}{F_{n-1} k-F_{n-2} h^{\prime}}
$$

where $x_{-1}=k, x_{0}=h$ and $F_{n-1}, F_{n-2}$ are the Fibonacci terms.

Proof: For $n=0$ the result holds. Now suppose that $n>0$ and that our assumption holds for $n-1, n-2$. That is;

$$
x_{n-2}=\frac{(-1)^{n-2} h k}{F_{n-3} k-F_{n-4} h}, x_{n-1}=\frac{(-1)^{n-1} h k}{F_{n-2} k-F_{n-3} h} \text {. }
$$


Now, it follows from Equation 7 that

$$
\begin{aligned}
x_{n} & =\frac{x_{n-1} x_{n-2}}{x_{n-1}-x_{n-2}}=\frac{\left(\frac{(-1)^{n-1} h k}{F_{n-2} k-F_{n-3} h}\right)\left(\frac{(-1)^{n-2} h k}{F_{n-3} k-F_{n-4} h}\right)}{\left(\frac{(-1)^{n-1} h k}{F_{n-2} k-F_{n-3} h}-\frac{(-1)^{n-2} h k}{F_{n-3} k-F_{n-4} h}\right)} \\
& =\frac{\left(\frac{(-1)^{n-1} h k}{F_{n-2} k-F_{n-3} h}\right)\left(\frac{-1}{F_{n-3} k-F_{n-4} h}\right)}{\left(\frac{1}{F_{n-2} k-F_{n-3} h}+\frac{1}{F_{n-3} k-F_{n-4} h}\right)}=\frac{(-1)^{n} h k}{\left(F_{n-2} k-F_{n-3} h+F_{n-3} k-F_{n-4} h\right)} \\
& =\frac{(-1)^{n} h k}{F_{n-1} k-F_{n-2} h} \cdot n
\end{aligned}
$$

Hence, the proof is completed.

For confirming the results of this section, we consider numerical example for $x_{-1}=$ 11, $x_{0}=4$ (see Figure 1), and for $x_{-1}=6, x_{0}=15$ (see Figure 2), since the solutions take the forms $\{6,-12,4,-3,1.714286,-1.090909, .6666667,-.4137931, .2553191, \ldots$.$\} ,$ $\{-60,10,-8.571428,4.615385,-3,1.818182,-1.132075, .6976744, \ldots\}$.

\subsection{Case (2)}

In this section we study the following special case of Equation 1

$$
x_{n+1}=\frac{x_{n-1} x_{n-2}}{x_{n-1}-x_{n-2}},
$$

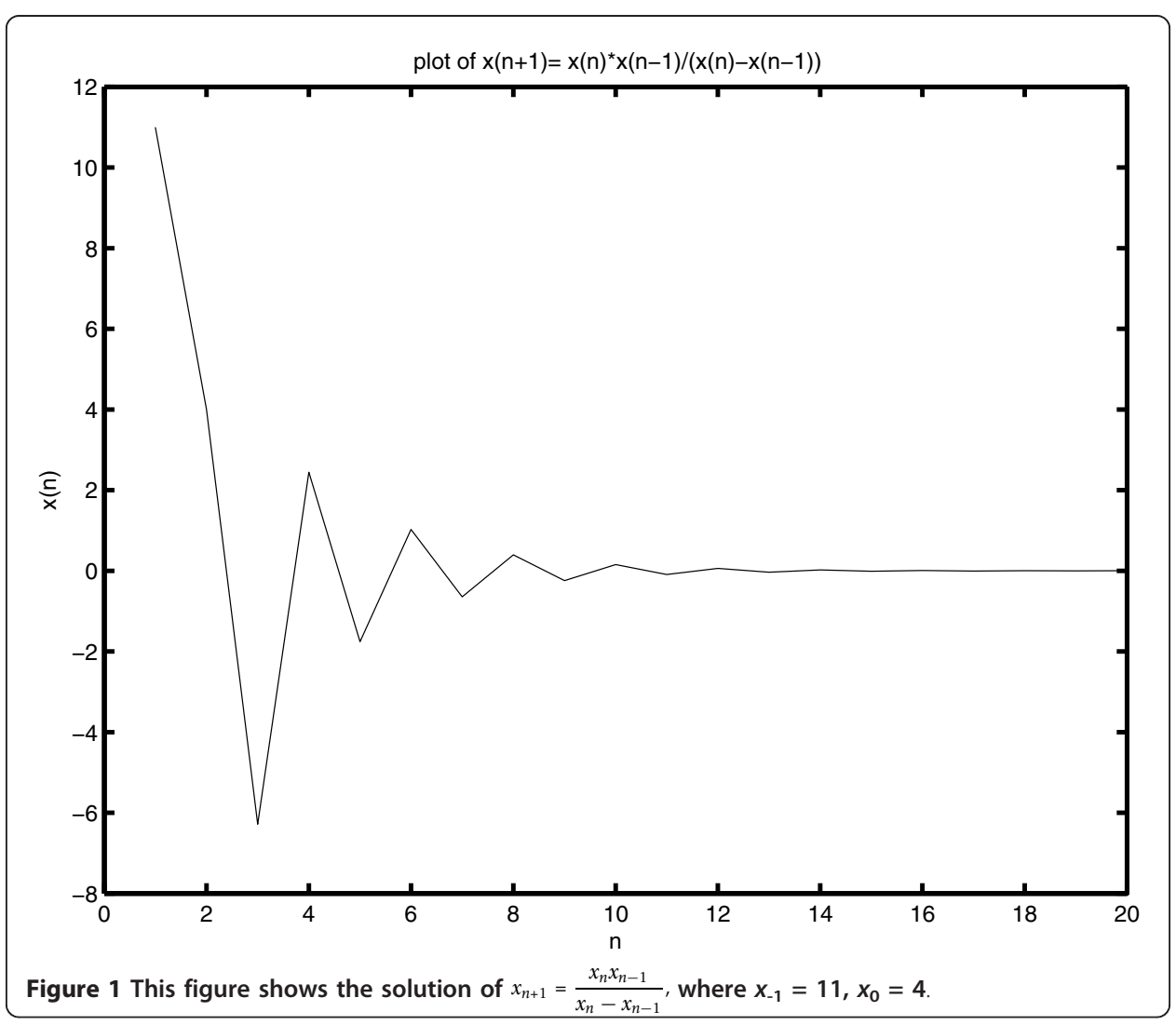




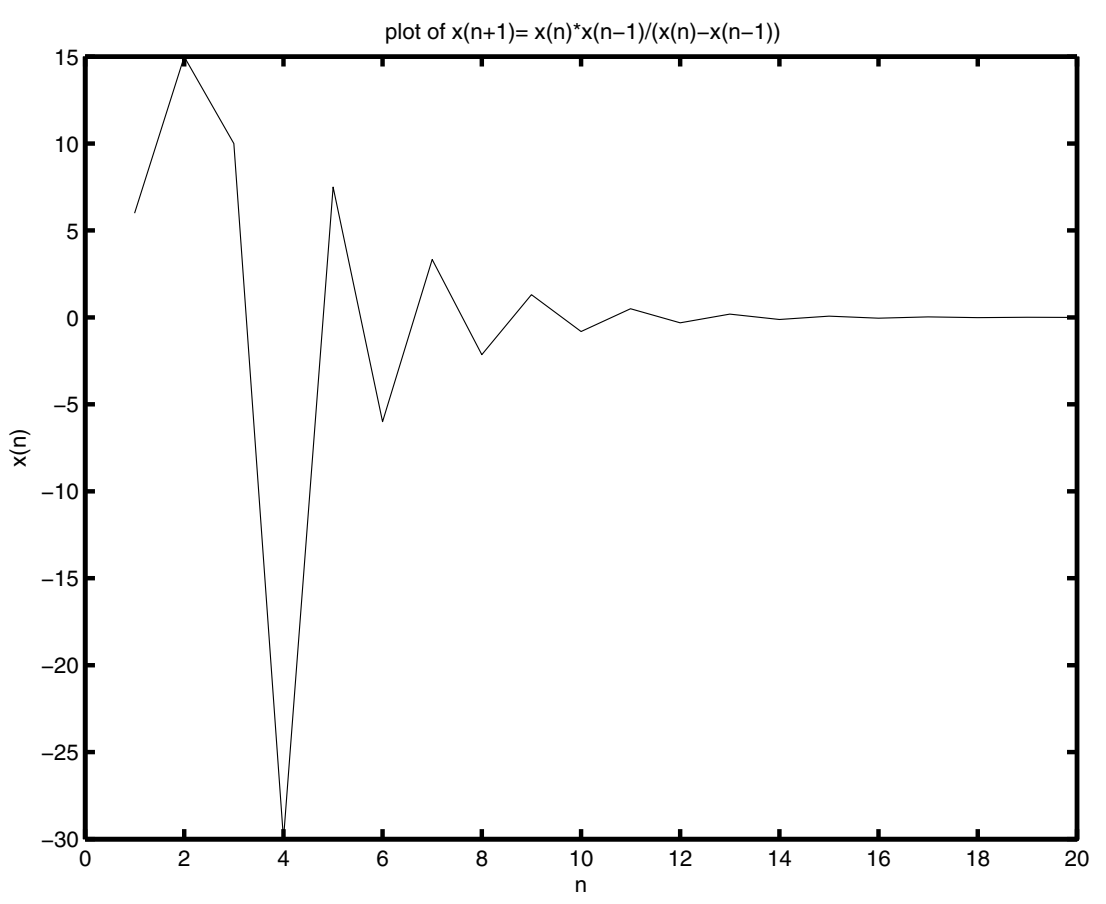

Figure 2 This figure shows the solution of $x_{n+1}=\frac{x_{n} x_{n-1}}{x_{n}-x_{n-1}}$, for $x_{-1}=6, x_{0}=15$

where the initial conditions $x_{-2}, x_{-1}, x_{0}$ are arbitrary positive real numbers.

\section{Theorem 6}

Let $\left\{x_{n}\right\}_{n=-2}^{\infty}$ be a solution of Equation 8. Then $x_{1}=\frac{r k}{k-r}$, for $n=1,2, \ldots$

$$
x_{n+1}=\frac{h k r}{g_{n-4} h k+g_{n-3} k r+g_{n-2} h r},
$$

where $x_{-2}=r, x_{-1}=k, x_{0}=h,\left\{g_{m}\right\}_{m=0}^{\infty}=\{1,-2,0,3,-2,-3, \ldots\}$, i.e., $g_{m}=g_{m-2}+$ $g_{m-3}, m \geq 0, g_{-3}=0, g_{-2}=-1, g_{-1}=1$.

Proof: For $n=1,2$ the result holds. Now suppose that $n>1$ and that our assumption holds for $n-1, n-2$. That is;

$$
x_{n-2}=\frac{h k r}{g_{n-7} h k+g_{n-6} k r+g_{n-5} h r} x_{n-1}=\frac{h k r}{g_{n-6} h k+g_{n-5} k r+g_{n-4} h r} \text {. Now, it follows }
$$

from Equation 8 that

$$
\begin{aligned}
x_{n+1} & =\frac{x_{n-1} x_{n-2}}{x_{n-1}-x_{n-2}} \\
& =\frac{\left(\frac{h k r}{g_{n-6} h k+g_{n-5} k r+g_{n-4} h r}\right)\left(\frac{h k r}{g_{n-7} h k+g_{n-6} k r+g_{n-5} h r}\right)}{\left(\frac{h k r}{g_{n-6} h k+g_{n-5} k r+g_{n-4} h r}-\frac{h k r}{g_{n-7} h k+g_{n-6} k r+g_{n-5} h r}\right)} \\
& =\frac{h k r}{\left(g_{n-7} h k+g_{n-6} k r+g_{n-5} h r-g_{n-6} h k+g_{n-5} k r+g_{n-4} h r\right)} \\
& =\frac{h k}{g_{n-4} h k+g_{n-3} k r+g_{n-2} h r} .
\end{aligned}
$$


Hence, the proof is completed.

Assume that $x_{-2}=8, x_{-1}=15, x_{0}=7$, then the solution will be $\{17.14286,-13.125$, $11.83099,7.433628,-6.222222,-20,3.387097,-9.032259, \ldots\}$ (see Figure 3).

The proof of following cases can be treated similarly.

\subsection{Case (3)}

Let $x_{-2}=r, x_{-1}=k, x_{0}=h, \prod_{i=0}^{-1} A_{i}=1$ and $F_{2 i-1}, F_{2 i}, F_{2 i+1}$ (where $i=0$ to $n$ ) are the Fibonacci terms. Then the solution of the difference equation

$$
x_{n+1}=\frac{x_{n-1} x_{n-2}}{x_{n}-x_{n-2}}
$$

is given by

$$
x_{2 n}=\frac{h \prod_{i=0}^{n-1}\left(F_{2 i-1} h-F_{2 i} r\right)}{\prod_{i=0}^{n-1}\left(F_{2 i+1} r-F_{2 i} h\right)}, x_{2 n+1}=\frac{k r \prod_{i=0}^{n-1}\left(F_{2 i+1} r-F_{2 i} h\right)}{\prod_{i=0}^{n}\left(F_{2 i-1} h-F_{2 i} r\right)}, n=0,1, \ldots .
$$

Figure 4 shows the solution when $x_{-2}=9, x_{-1}=12, x_{0}=17$.

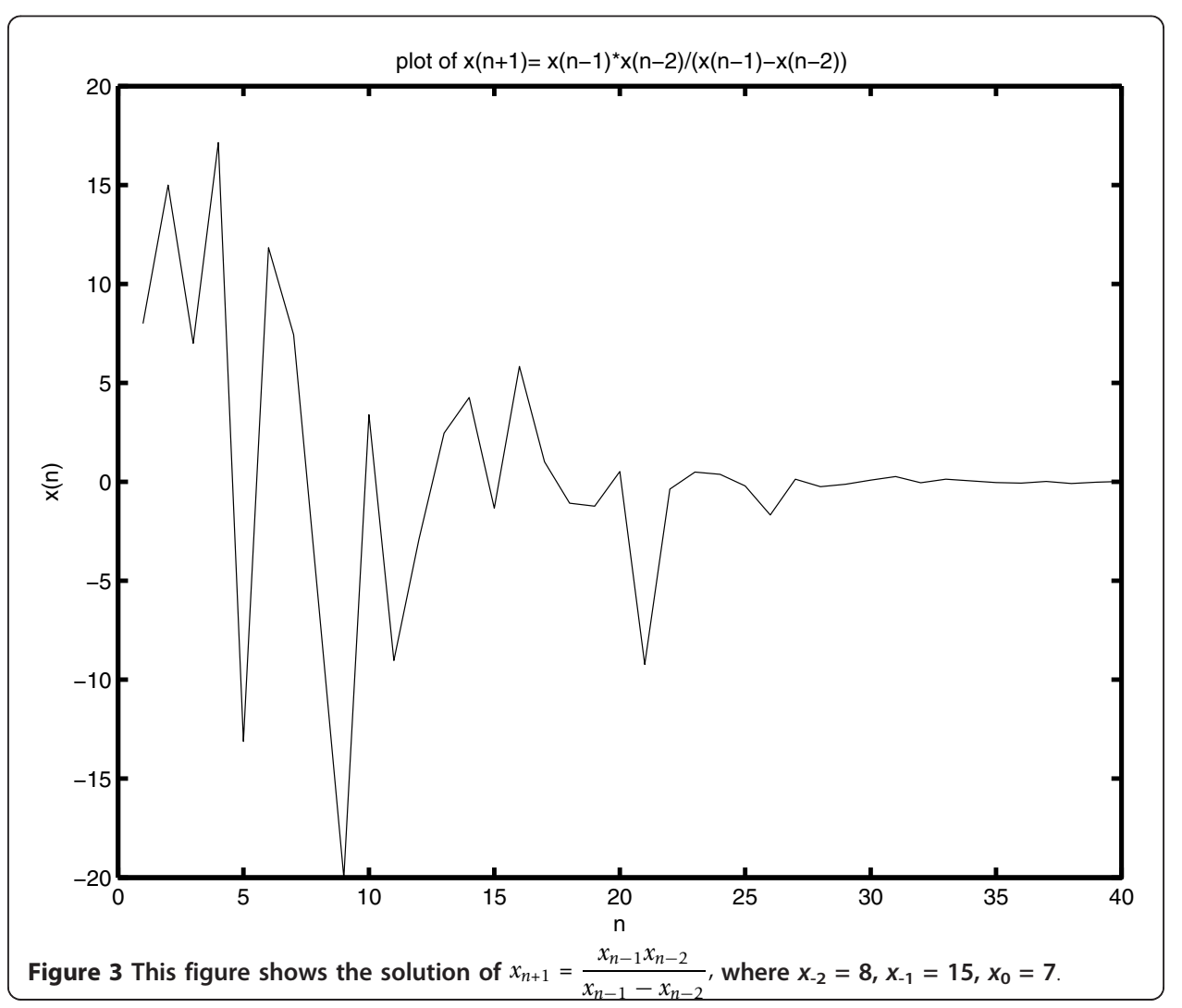




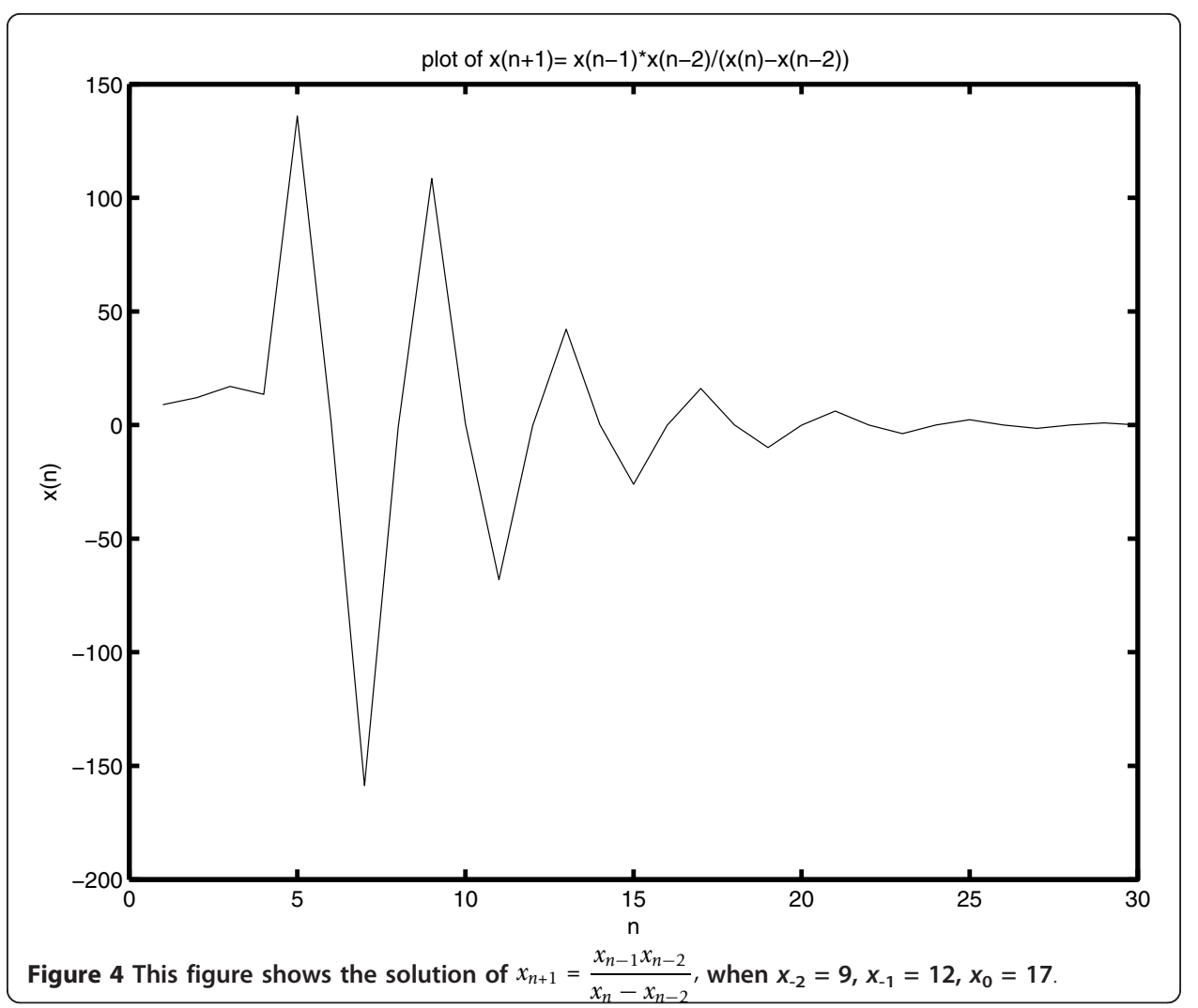

4.4 Case (4)

Let $x_{-2}=r, x_{-1}=k, x_{0}=h$. Then the solution of the following difference equation

$$
x_{n+1}=\frac{x_{n-1} x_{n}}{x_{n}-x_{n-2}}
$$

is given by

$$
x_{2 n-1}=\left(\frac{h}{h-r}\right)^{n} k, \quad x_{2 n}=\frac{h^{n+1}}{r^{n}}, n=0,1, \ldots .
$$

Figure 5 shows the solution when $x_{-2}=21, x_{-1}=6, x_{0}=3$.

\subsection{Case (5)}

Let $x_{-2}=r, x_{-1}=k, x_{0}=h$. Then the solution of the following difference equation

$$
x_{n+1}=\frac{x_{n-1} x_{n}}{x_{n-1}-x_{n-2}}
$$

is given by

$$
\begin{aligned}
& x_{4 n}=\frac{h(h k)^{2 n}}{(r k(h-k)(k-r))^{n}}, x_{4 n+1}=\frac{(h k)^{2 n+1}}{(r k(h-k))^{n}(k-r)^{n+1}}, \\
& x_{4 n+2}=\frac{h(h k)^{2 n+1}}{((h-k)(k-r))^{n+1}(r k)^{n}}, x_{4 n+3}=\frac{(h k)^{2 n}}{(r(h-k)(k-r))^{n+1} k^{n}}, n=0,1, \ldots .
\end{aligned}
$$

Figure 6 shows the solution when $x_{-2}=9, x_{-1}=5, x_{0}=4$. 

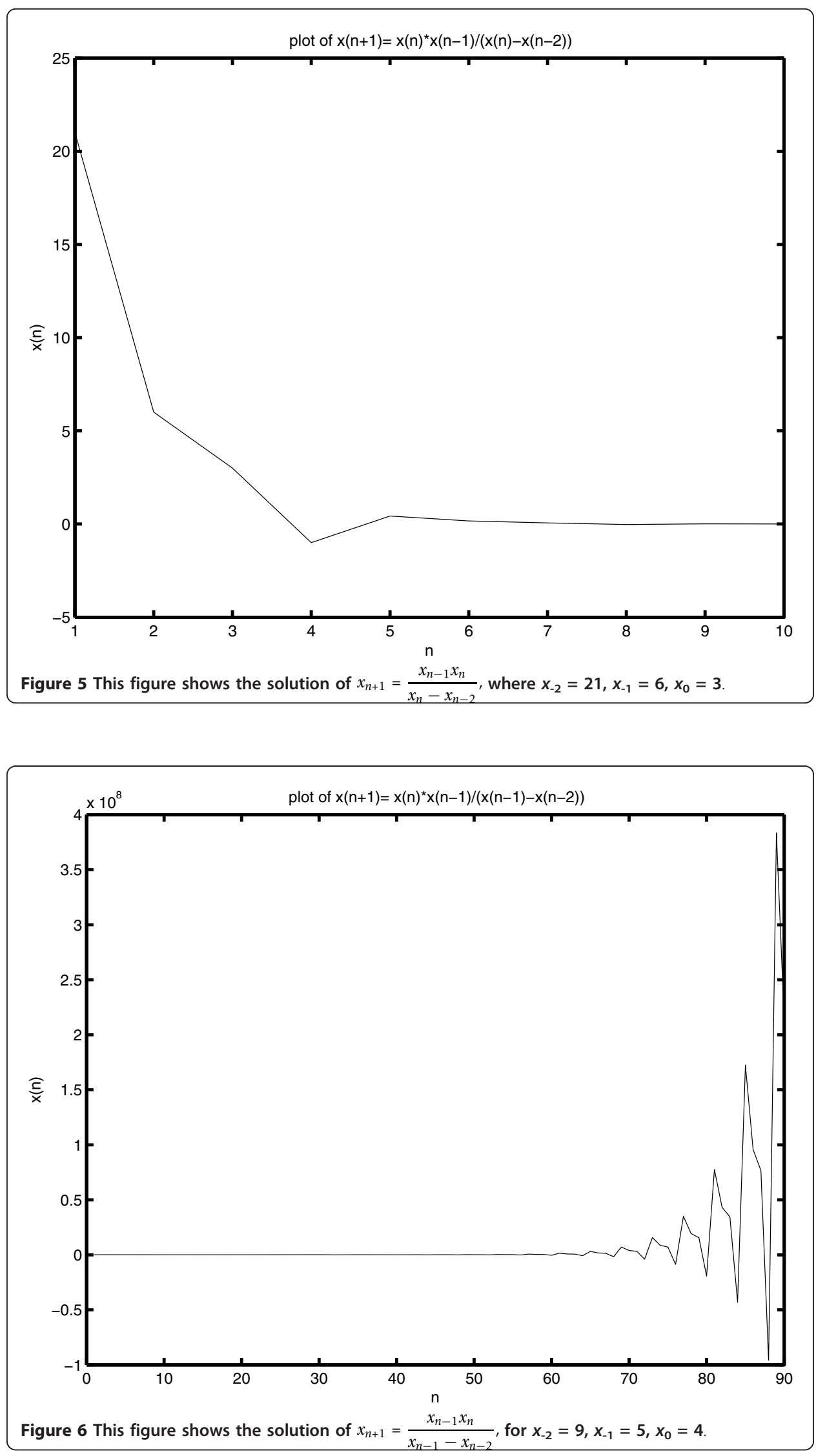
Figure 7 shows the solution when $x_{-2}=.9, x_{-1}=5, x_{0}=.4$.

\subsection{Case (6)}

Let $x_{-2}=r, x_{-1}=k, x_{0}=h$, Then the solution of the following difference equation

$$
x_{n+1}=\frac{x_{n-2} x_{n}}{x_{n}-x_{n-2}}
$$

is given by

$$
x_{n}=\frac{h k r}{u_{n-3} h r+u_{n-2} h k+u_{n-1} k r}, n=0,1, \ldots,
$$

Where $\left\{u_{m}\right\}_{m=0}^{\infty}=\{-1,1,0,-1,2,-2,1,1,-3, \ldots\}$ i. e. $u_{m}=u_{m-1}-u_{m-3}, m \geq 0, u_{-}$ $3=0, u_{-2}=0, u_{-1}=1$.

Figure 8 shows the solution when $x_{-2}=11, x_{-1}=6, x_{0}=17$.

\subsection{Case (7)}

Let $x_{-2}=r, x_{-1}=k, x_{0}=h$ and $\mathrm{F}_{n-1} \mathrm{~F}_{, n-2}, F_{n}$ are the Fibonacci terms.

Then the solution of the following difference equation

$$
x_{n+1}=\frac{x_{n-2} x_{n}}{x_{n-1}-x_{n-2}}
$$

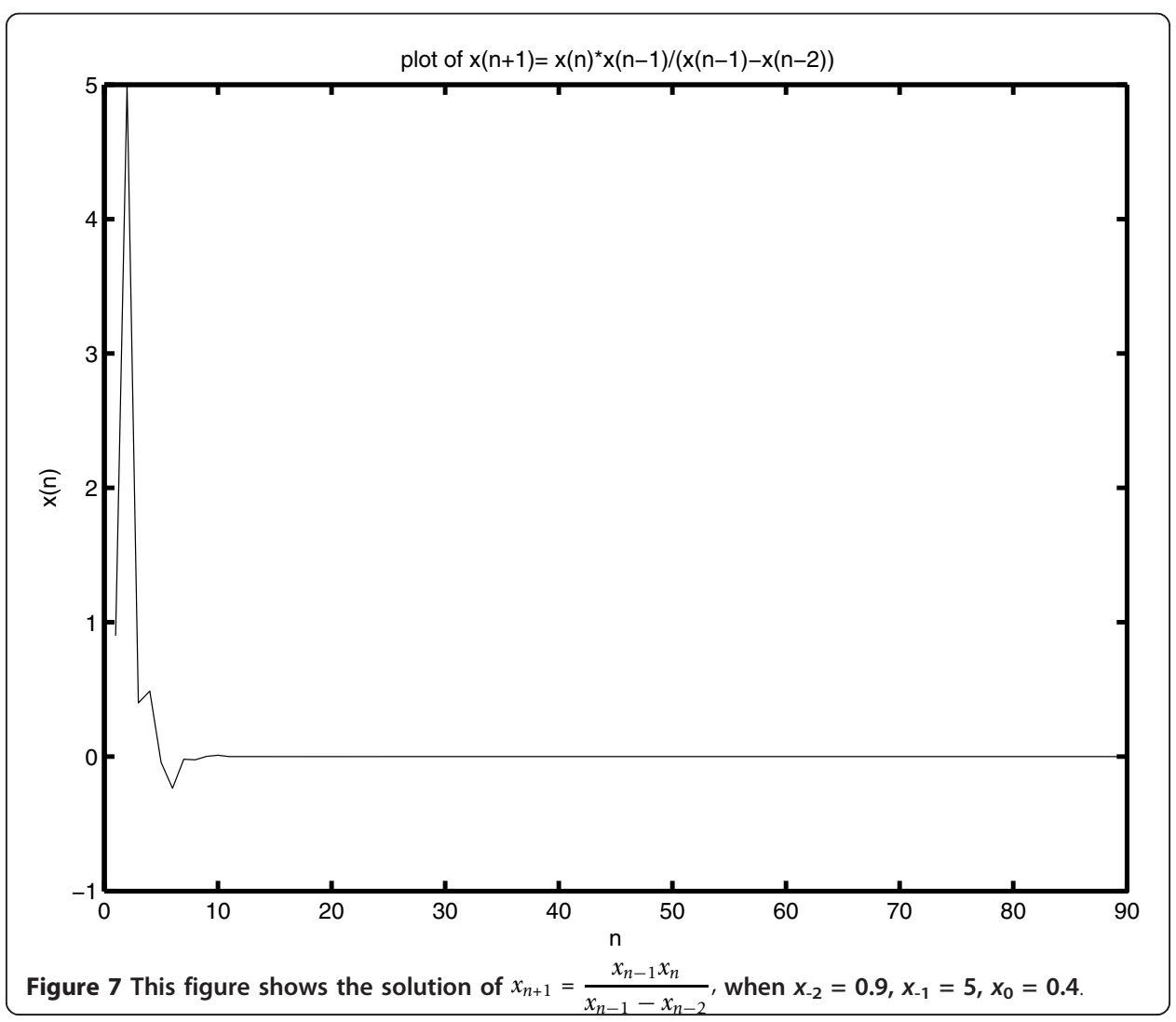



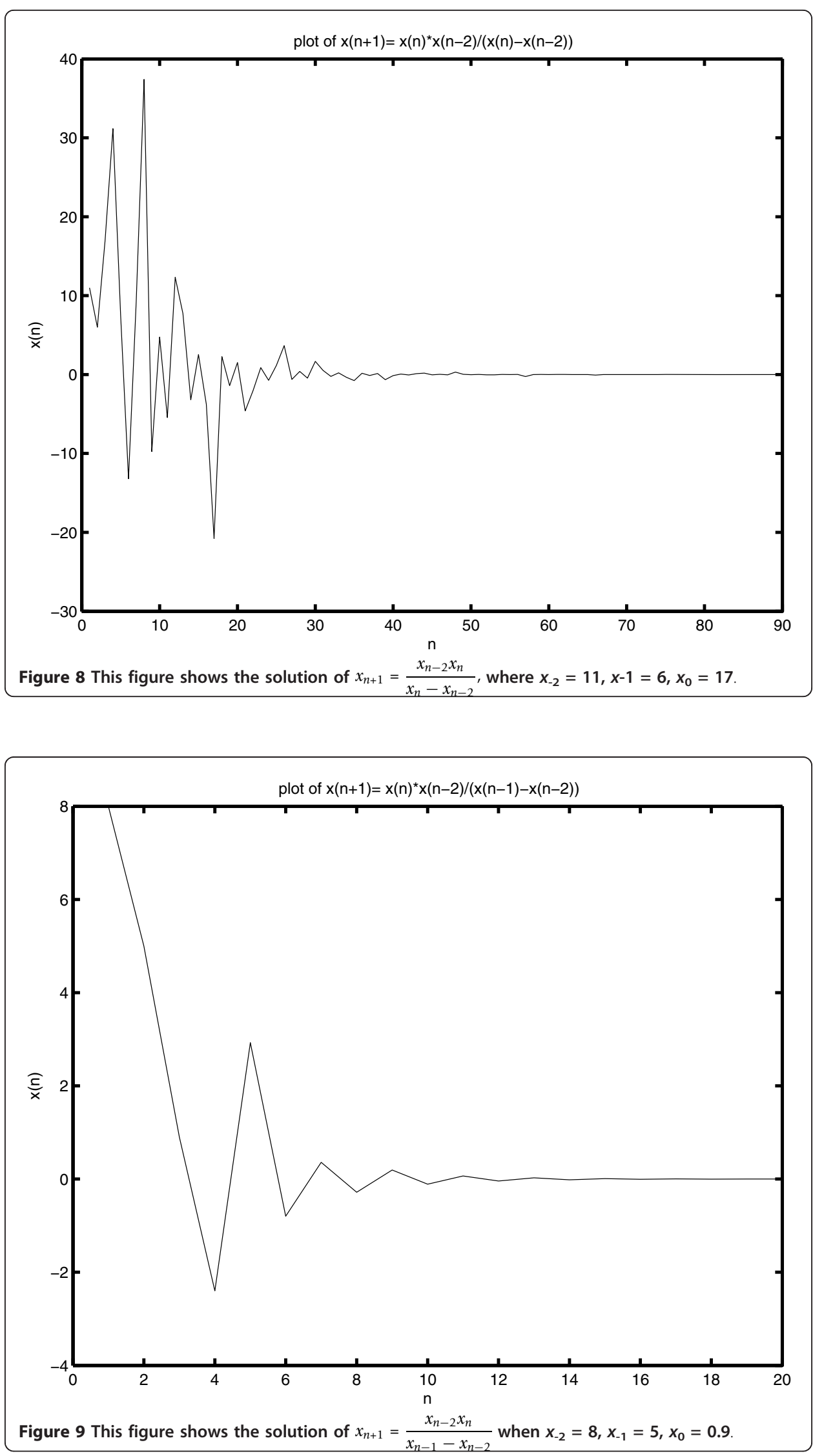
is given by

$$
\begin{aligned}
& x_{2 n}=\frac{h k r}{\left(F_{n-2} k-F_{n-1} r\right)\left(F_{n-2} h-F_{n-1} k\right)}, \\
& x_{2 n+1}=\frac{h k r}{\left(F_{n-1} k-F_{n} r\right)\left(F_{n-2} h-F_{n-1} k\right)}, n=0,1, \ldots .
\end{aligned}
$$

Figure 9 shows the solution when $x_{-2}=8, x_{-1}=5, x_{0}=0.9$.

\section{Author details}

${ }^{1}$ Mathematics Department, Faculty of Science, Mansoura University, Mansoura 35516, Egypt ${ }^{2}$ Department of Mathematics, Faculty of Science \& Art in Rabigh, King AbdulAziz University, Rabigh 21911, Saudi Arabia ${ }^{3}$ Mathematics Department, Faculty of Science, King AbdulAziz University, P. O. Box 80203, Jeddah 21589, Saudi Arabia ${ }^{4}$ Permanent address: Mathematics Department, Faculty of Science, Mansoura University, Mansoura 35516, Egypt

\section{Authors' contributions}

EMEla investigated the behavior of the solutions, HAE-M found the solutions of the special cases and EMEls carried out the theoretical proof and gave the examples. All authors read and approved the final manuscript.

\section{Competing interests}

The authors declare that they have no competing interests.

Received: 5 April 2011 Accepted: 23 August 2011 Published: 23 August 2011

\section{References}

1. Kulenovic MRS, aa, Ladas, G: Dynamics of Second Order Rational Difference Equations with Open Problems and Conjectures. Chapman \& Hall/CRC Press, Boca Raton, FL. (2001)

2. Kocic, VL, Ladas, G: Global Behavior of Nonlinear Difference Equations of Higher Order with Applications. Kluwer Academic Publishers, Dordrecht (1993)

3. Mickens, RE: Difference Equations. Van Nostrand Reinhold Comp, New York. (1987)

4. Mackey, MC, Glass, L: Oscillation and chaos in physiological control system. Science. 197, 287-289 (1977). doi:10.1126/ science. 267326 5. Elabbasy, EM, El-Metwally, H, Elsayed, EM: On the difference equation $x_{n+1}=a x_{n}-\frac{b x_{n}}{c x_{n}-d x_{n-1}}$. Adv Differ Equ 1-10
(2006). Article ID 82579

6. Elabbasy, EM, El-Metwally, H, Elsayed, EM: On the difference equations $x_{n+1}=\frac{\alpha x_{n-k}}{\beta+\gamma \Pi_{i=0}^{k} x_{n-i}}$. J Conc Appl Math. 5(2), 101-113 (2007)

7. Elabbasy, EM, El-Metwally, H, Elsayed, EM: Global attractivity and periodic character of a fractional difference equation of order three. Yoko-hama Math J. 53, 89-100 (2007)

8. El-Metwally, H, Grove, EA, Ladas, G, Levins, R, Radin, M: On the difference equation $x_{n+1}=\alpha+\beta x_{n-1} e^{-x_{n}}$.. Nonlinear Anal: Theory Methods Appl. 47(7), 4623-4634 (2003)

9. Yang, X, Su, W, Chen, B, Megson, GM, Evans, DJ: On the recursive Sequence $x_{n+1}=\frac{a x_{n-1}+b x_{n-2}}{c+d x_{n-1} x_{n-2}}$. Appl Math Comput. 162, 1485-1497 (2005). doi:10.1016/j.amc.2004.03.023

10. Cinar, C: On the positive solutions of the difference equation $x_{n+1}=\frac{x_{n-1}}{1+a x_{n} x_{n-1}}$. Appl Math Comput. 158(3), 809-812 (2004). doi:10.1016/j.amc.2003.08.140

11. Cinar, C: On the positive solutions of the difference equation $x_{n+1}=\frac{x_{n-1}}{-1+a x_{n} x_{n-1}}$. Appl Math Comput. 158(3), 793-797 (2004). doi:10.1016/j.amc.2003.08.139

12. Aloqeili, M: Dynamics of a rational difference equation. Appl Math Comput. 176(2), 768-774 (2006). doi:10.1016/j. amc.2005.10.024

13. Yalçinkaya, I: On the difference equation $x_{n+1}=\alpha+\frac{x_{n-m}}{x_{n}^{k}}$. Discrete Dyn Nat Soc (2008). Article ID 805460, 8

14. Agarwal, R: Difference Equations and Inequalities. Theory, Methods and Applications. Marcel Dekker Inc., New York (1992)

15. Agarwal, RP, Elsayed, EM: Periodicity and stability of solutions of higher order rational difference equation. Adv Stud Contemp Math. 17(2), 181-201 (2008)

16. Agarwal, RP, Zhang, W: Periodic solutions of difference equations with general periodicity. Comput Math Appl. 42 , 719-727 (2001). doi:10.1016/50898-1221(01)00191-2

17. Elabbasy, EM, Elsayed, EM: On the global attractivity of difference equation of higher order. Carpathian J Math. 24(2), 45-53 (2008)

18. Elabbasy, EM, Elsayed, EM: Dynamics of a rational difference equation. Chin Ann Math Ser B. 30B(2), 187-198 (2009)

19. El-Metwally, H, Grove, EA, Ladas, G, McGrath, LC: On the difference equation $y_{n+1}=\frac{y_{n-(2 k+1)}+p}{y_{n-(2 k+1)}+q y_{n-2 l}}$. Proceedings of the 6th ICDE, Taylor and Francis, London (2004)

20. Elsayed, EM: Qualitative behavior of difference equation of order three. Acta Sci Math (Szeged). 75(1-2), 113-129 (2009)

21. Elsayed, EM: Qualitative behavior of s rational recursive sequence. Indagat Math. 19(2), 189-201 (2008). doi:10.1016/ S0019-3577(09)00004-4

22. Elsayed, EM: On the Global attractivity and the solution of recursive sequence. Stud Sci Math Hung. 47(3), 401-418 (2010) 
23. Elsayed, EM: Qualitative properties for a fourth order rational difference equation. Acta Appl Math. 110(2), 589-604 (2010). doi:10.1007/s10440-009-9463-z

24. Wang, C, Wang, S, Yan, X: Global asymptotic stability of 3-species mutualism models with diffusion and delay effects. Discrete Dyn Nat Sci 20 (2009). Article ID 317298

25. Wang, C, Gong, F, Wang, S, Li, L, Shi, Q: Asymptotic behavior of equilibrium point for a class of nonlinear difference equation. Adv Diff Equ 8, : (2009). Article ID 214309

26. Yalçinkaya, I, Iricanin, BD, Cinar, C: On a max-type difference equation. Discrete Dyn. Nat Soc (2007). Article ID 47264, 10

27. Yalçinkaya, I: On the global asymptotic stability of a second-order system of difference equations. Discrete Dyn Nat Soc (2008). Article ID 860152, 12

28. Yalçinkaya, I: On the global asymptotic behavior of a system of two nonlinear difference equations. ARS Combinatoria. 95, 151-159 (2010) 29. Zayed, EME, El-Moneam, MA: On the rational recursive sequence $x_{n+1}=a x_{n}-\frac{b x_{n}}{c x_{n}-d x_{n-k}}$.. Commun Appl Nonlinear
Anal. 15, 47-57 (2008)

doi:10.1186/1687-1847-2011-28

Cite this article as: Elabbasy et al:: Global behavior of the solutions of some difference equations. Advances in

Difference Equations 2011 2011:28.

\section{Submit your manuscript to a SpringerOpen ${ }^{\circ}$} journal and benefit from:

- Convenient online submission

- Rigorous peer review

- Immediate publication on acceptance

- Open access: articles freely available online

- High visibility within the field

- Retaining the copyright to your article

Submit your next manuscript at $\gg$ springeropen.com 